\title{
VERTICAL VELOCITIES ASSOCIATED WITH PLAGE REGION MAGNETIC FIELDS
}

\author{
R. G. GIOVANELLI and J. V. RAMSAY \\ CSIRO Division of Physics, National Standards Laboratory, Sydney, Australia 2008
}

The Culgoora magnetograph (Ramsay et al., 1970) produces simultaneous filtergrams in opposite circular polarizations at a wavelength selected by a filter bandwidth $0.005 \mathrm{~nm}$ (Ramsay et al., 1970). In the blue wing of the $610.27 \mathrm{~nm}$ line of CaI, regions of magnetic fields in strong or weak plages are very obvious in one or other polarization, depending on polarity, even before subtraction; in one polarization they are bright, but almost invisible in the other. They are more difficult to discern at equal intervals from the line centre in the other wing (Figure 1). When subtractions are carried out to yield magnetograms of the same sense, the two magnetograms from opposite wings give results which appear to be much the same. An example is shown in Figure 2. Similar results are obtained over a wide range of positions in the wings of the $610.27 \mathrm{~nm}$ line.

Our observations have involved many differing states of adjustment of the filter for which the instrumental line profile must have varied over wide limits without any substantial change in the relative appearances of the red- and blue-wing filtergrams. Rotation of the $\lambda / 4$ plate through $90^{\circ}$ has no significant effect beyond a reversal of the polarized filtergrams. Hence we conclude that the asymmetry in the appearances of the filtergrams is not due to the instrument but is of solar origin. We interpret it as due to a more-or-less uniform inwards velocity in magnetic regions.

The $610.27 \mathrm{~nm}$ absorption line is not as deep in magnetic as in non-magnetic regions, a result first found by Sheeley (1967) and Chapman and Sheeley (1968). Figure 3 shows a schematic line profile in the absence of magnetic field together with the combined effects of a longitudinal Zeeman splitting and a uniform Doppler shift to the red in a magnetic region. Filtergrams obtained in the blue wing will show magnetic regions much brighter than the background in one polarization, and of reduced contrast in the other. In the red wing, the maximum intensity difference from background is less than in the blue wing. However the intensity differences between the two polarizations are identical for the two wings.

A non-uniform vertical velocity would have the effect of making one wing of the line steeper than the other. This would cause magnetograms in one wing to show magnetic fields with greater contrast than in the other. Whilst magnetograms in opposite wings are not always identical, there does not appear to be any systematic difference, nor have we found any differences that could not be explained by seeing or the state of instrumental adjustment. We therefore conclude that the vertical velocity is more-orless uniform.

There is another way of demonstrating and measuring downwards velocities in magnetic regions. The filter, consisting of a series of interferometers, transmits a pass 


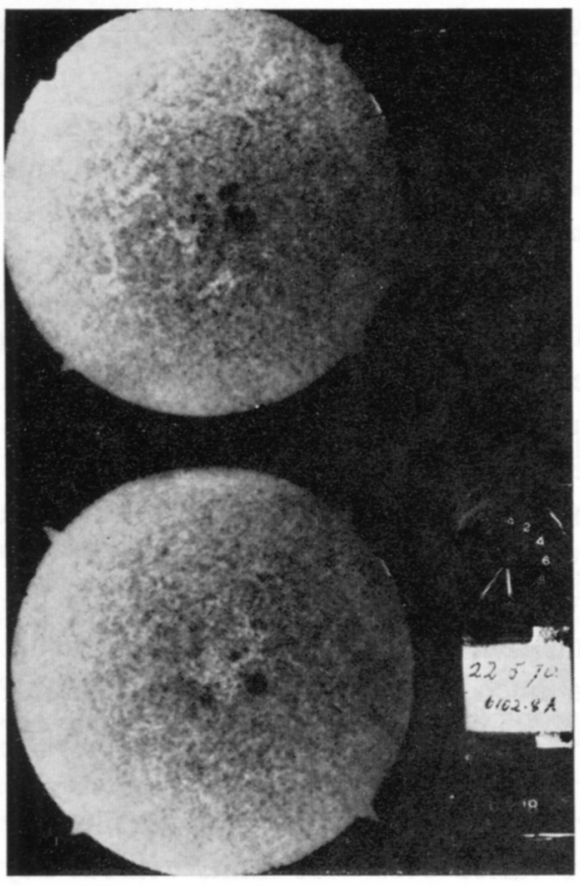

(a)

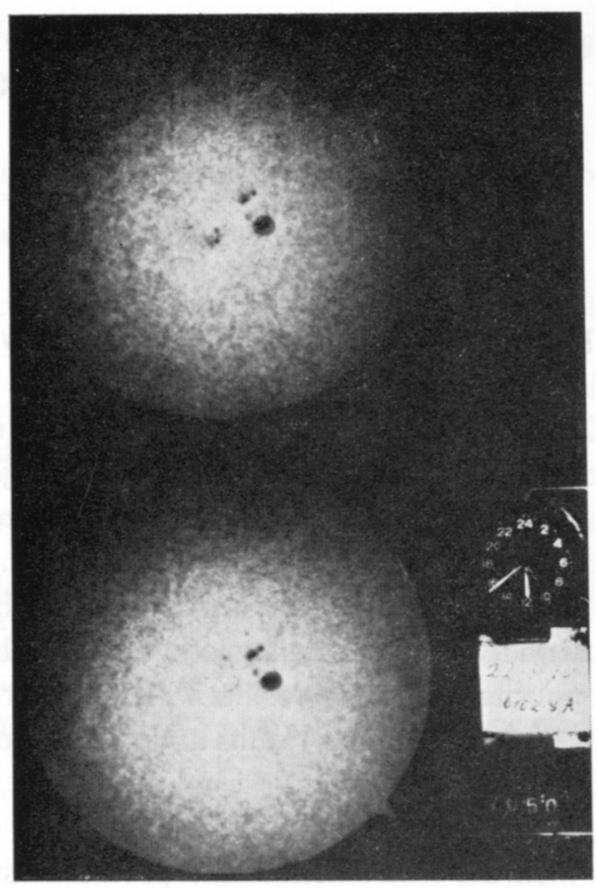

(b)

Fig. 1. Filtergrams in the CaI $610.27 \mathrm{~nm}$ absorption line, blue wing (a) and red wing (b). The two upper photographs are in one circular polarization, the two lower in the other.

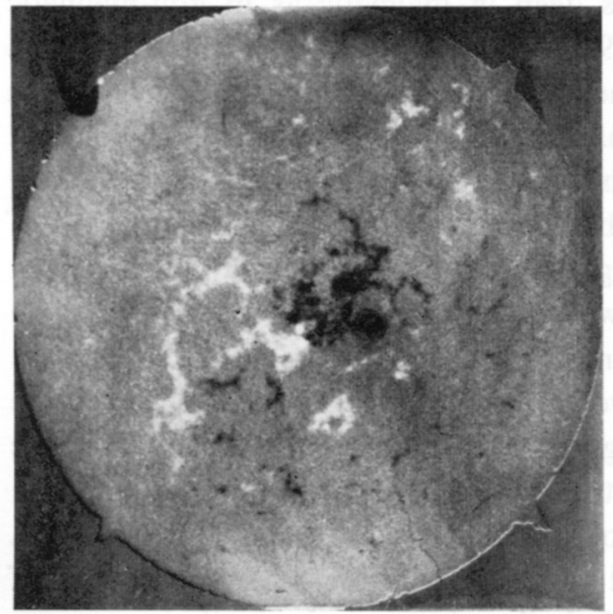

(a)

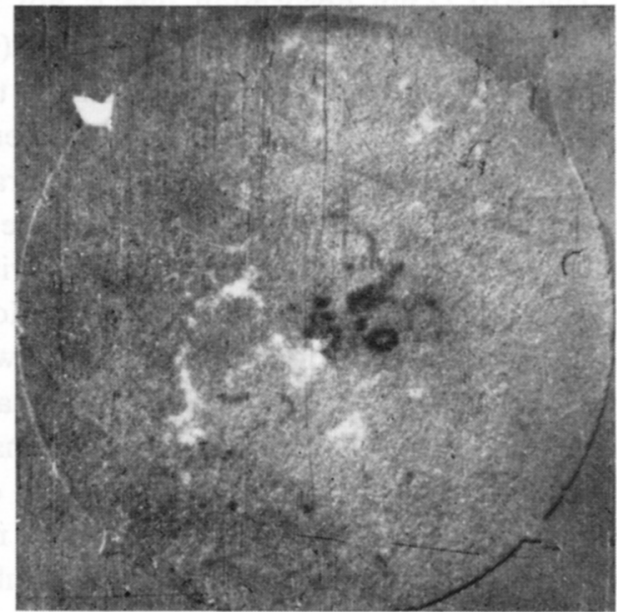

(b)

Fig. 2. Magnetograms obtained from filtergrams in Figure 1. (a) is from the blue wing, (b) from the red wing. 
band whose peak intensity varies in wavelength towards the blue by $0.005 \mathrm{~nm}$ from the centre of the field to the edge, the wavelength shift being proportional to the square of the distance from the centre. Thus a filtergram obtained with the centre of the field $0.0025 \mathrm{~nm}$ to the red of line centre has the edge of the field at $0.0025 \mathrm{~nm}$ to the blue, while at 0.707 of the radius from centre to edge there is a minimum intensity corresponding to line centre (Figure 4c). If two such filtergrams obtained in opposite circular polarizations are subtracted, magnetic fields will be seen near the

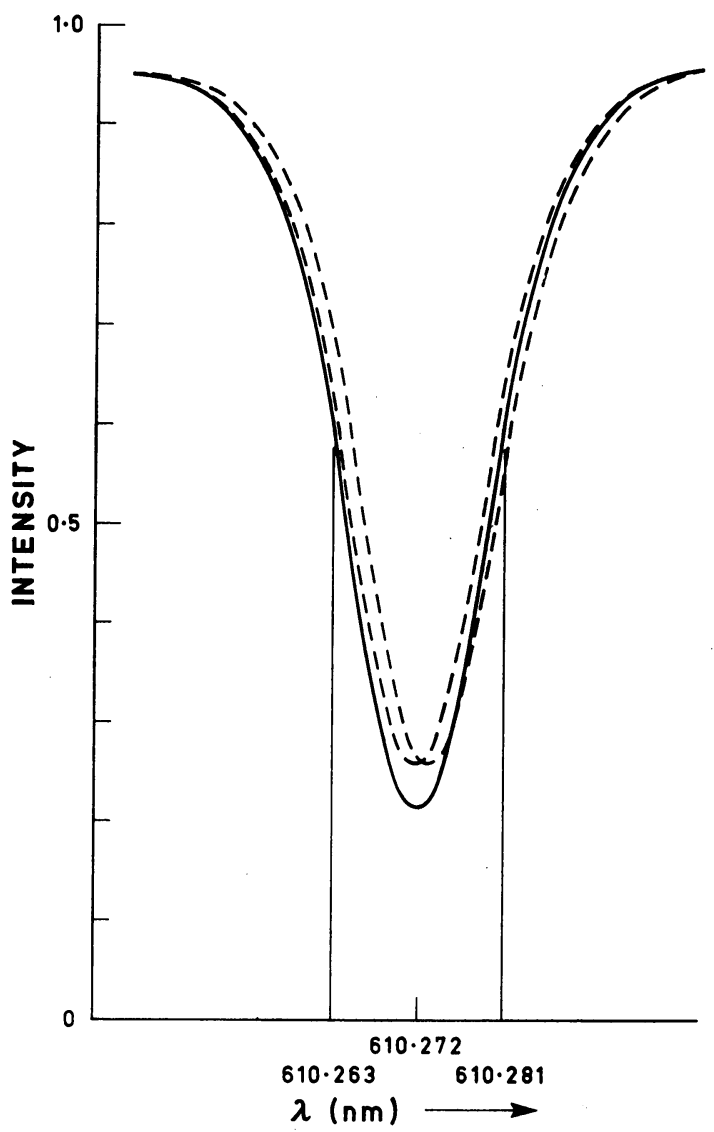

Fig. 3. Schematic line profile in a non-magnetic region (deepest core), and Doppler-shifted Zeeman components in a magnetic region.

centre of the picture in the same sense as in the red wing. Near the edge, they appear in the same sense as in the blue wing, while at the line centre of the magnetic region they are invisible. If the magnetic region has a Doppler shift, the reversal in sense does not occur exactly at the zone corresponding to the normal line centre. Figure 4 shows the effect, in which the reversal occurs at $0.0010 \mathrm{~nm}$ to the red of line centre (i.e., closer to the centre of the field), corresponding to a downwards velocity of $0.5 \mathrm{~km} \mathrm{~s}^{-1}$. This result is not affected systematically by seeing. 


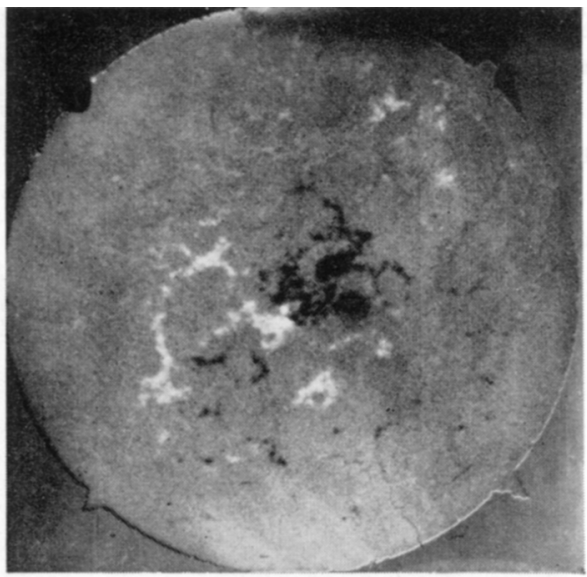

(a)

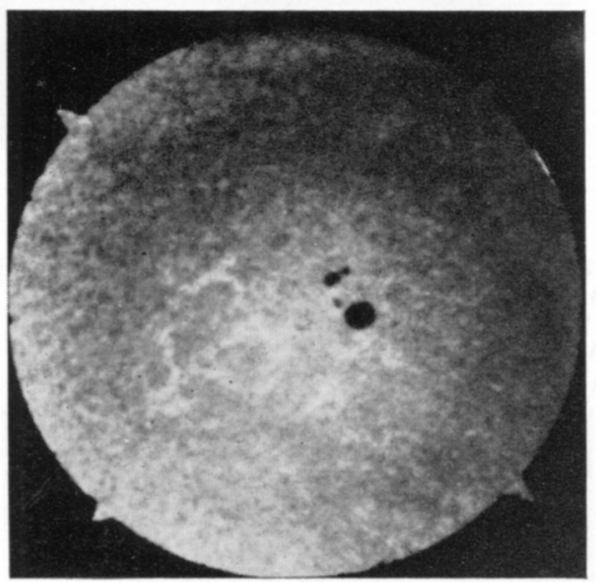

(c)

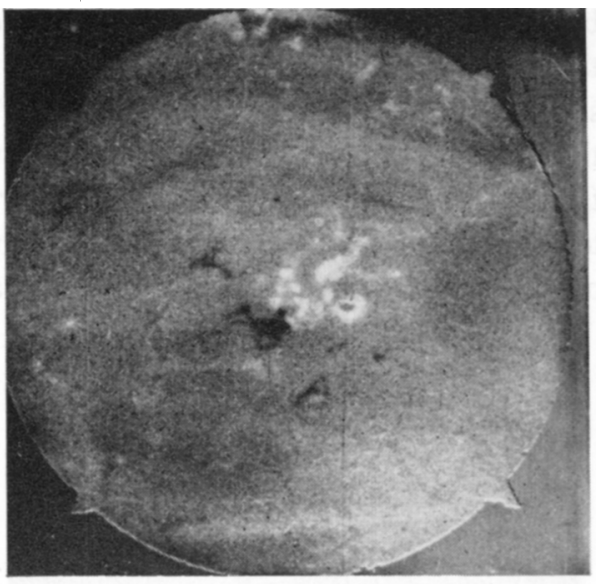

(b)

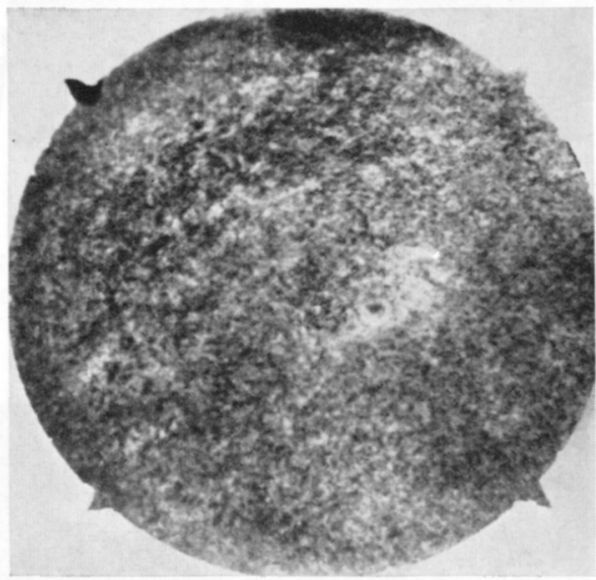

(d)

Fig. 4. (a) Magnetogram in CaI $610.27 \mathrm{~nm}$ absorption line, blue wing; (b) magnetogram near line centre; (c) one of the filtergrams from which (b) was derived. Note the dark ring in (c) corresponding to line centre. The corresponding velocity field with dark denoting a downwards velocity is shown in (d).

Both methods yield velocities in the same sense (inwards) and of the same magnitude.

When we compare these results with velocities observed in non-magnetic regions, an important difference emerges. As Leighton (1962) has shown for many lines including $610.27 \mathrm{~nm}$, there is a strong correlation between intensity at a given interval from the line centre and velocity as measured at that part of the line profile, such that the brighter (and hence hotter) regions are moving upwards. But where there are 
magnetic fields, the monochromatic intensity is greater and material is moving downwards!

Velocity photographs obtained by appropriate subtraction of filtergrams from opposite wings do not show an obvious pattern corresponding to the magnetic field, though comparisons with the magnetograms in a blink comparator show, in conformity with the results described above, that in magnetic regions the velocities are downward (Figure 4d). Thus the magnetic field does not appear to be the cause of the associated downward velocities. Beckers and Schröter (1968) have previously stated that magnetic regions are almost always associated with downward velocities, the rms value being about the same as in the undisturbed photosphere. Our results are in agreement with theirs.

\section{References}

Beckers, J. M. and Schröter, E. H.: 1968, Solar Phys. 4, 142.

Chapman G. A. and Sheeley, N. R.: 1968, Solar Phys. 5, 442.

Leighton, R. B.: 1962, Astrophys. J. 135, 474.

Ramsay, J. V., Kobler, H., and Mugridge, E. G. V.: 1970, Solar Phys. 12, 492.

Ramsay, J. V., Giovanelli, R. G., and Gillett, H. R.: 1971, this volume, p. 24.

Sheeley, N. R.: 1967, Solar Phys. 1, 171. 